\title{
Editorial
}

\section{Evidence-Based Myopia Control: An Overview}

\author{
Frank Spors ${ }^{\star}$ and Jie Shen \\ College of Optometry, Western University of Health Sciences, Pomona, California, USA
}

*Corresponding author: Dr. Frank Spors, College of Optometry, Western University of Health Sciences, Pomona, California, USA, Tel: +1 9097063883; E-mail: fspors@westernu.edu

Received Date: 17 November, 2017; Accepted Date: 29 November, 2017; Published Date: 14 December, 2017

\section{The Need for Controlling Myopia Pro- gression}

Myopia is a common ocular disorder which usually involves an increased axial length of the eye.High myopia of more than -6.00 diopters is associated with a higher risk of ocular diseases [1]. The prevalence rates of myopia are rising rapidly worldwide. Currently, every 1 out of 3 adults in the United States is myopic [2]. In some Asian countries, $60 \%-80 \%$ of young adults are myopic [3]. Some reports suggest that close to half of the world's population may be myopic by 2050 . Of those, about $10 \%$ may have high myopia, which is linked to pathological myopia [4].

The rising prevalence rate of childhood myopia is thought to be linked to changes in the everyday visual environment and has become of considerable concern for parents and healthcare providers. Therefore efforts to control myopia progression are escalating and prevention of myopia has become an international public health priority [5].

\section{Evidence-Based Strategies for Myopia Control}

Several strategies, which have been proposed for retarding myopia progression in the past, were either not found to be effective at all, or the effects were so small that they are not recommended as a routine prescription for myopic children. Among these are under corrected single vision spectacle lenses, single vision Rigid Gas-Permeable (RGP) contact lenses in alignment fit, single vision soft contact lenses, and administration of timolol $(0.25 \%)$ eye drops, which all were ineffective. Weak myopia control effects were demonstrated by bifocal spectacle lenses, multifocal spectacle lenses, and peripheral defocus modifying spectacle lenses. Prismatic bifocal spectacle lenses showed moderate effects.

An increase in outdoor activities seems to reduce the onset of myopia and subsequently has a weak impact on myopia progression [6].
Other myopia control studies show promising results, with the most effective methods being orthokeratology, soft bifocal contact lenses, and pharmacologic, antimuscarinic agents, which are applied as eye drops [7].

Assessing various myopia control strategies, a robust number of Randomized Controlled Trials (RCT) have been conducted in recent years. An RCT is considered the gold standard to test the efficacy or effectiveness of a particular medical intervention. This article briefly summarizes the results of recently conducted myopia control RCTs.

A meta-analysis, published 2016 in the journal Ophthalmology, analyzed 30 RCT interventions for myopia control in children with treatment duration of at least 1 year and compared their efficacy [8]. The analyzed studies included 4 main types of interventions: spectacle lenses (13 RCT), contact lenses (9 RCT), pharmacologic agents (7 RCT), and more outdoor activities (1 $\mathrm{RCT}$ ). When monitoring myopia progression, ideally both refraction and axial length were assessed. While this was done by 19 studies, 9 studies only reported refraction, and 2 studies reported only axial length. A total of 5422 eyes were involved in the analysis. The authors labeled the strength of a particular interventive strategy for controlling myopia as strong, moderate, weak, or ineffective in direct comparison with single vision spectacle lenses or placebo treatment as shown in table 1. A summary of the results is provided in the following sections.

\begin{tabular}{|c|c|c|}
\hline $\begin{array}{c}\text { Myopia } \\
\text { control effect }\end{array}$ & $\begin{array}{c}\text { Refraction } \\
\text { change per year }\end{array}$ & $\begin{array}{c}\text { Axial length } \\
\text { change per year }\end{array}$ \\
\hline Strong & More than 0.50 D & More than $-0.18 \mathrm{~mm}$ \\
\hline Moderate & $0.25 \mathrm{D}$ to 0.50 D & $-0.09 \mathrm{~mm}$ to $-0.18 \mathrm{~mm}$ \\
\hline Weak & 0.00 D to 0.25 D & $0.00 \mathrm{~mm}$ to $-0.09 \mathrm{~mm}$ \\
\hline Ineffective & Negative D & Positive $\mathrm{mm}$ \\
\hline
\end{tabular}

Table 1: Myopia control effect labeling based on changes of refraction and axial length per year, compared to single vision lenses or placebo treatment. 


\section{Spectacle Lenses}

The efficacy of spectacle lenses for myopia control is quite variable, ranging from moderately effective to ineffective, depending on the type of spectacle lens.

Prismatic bifocal spectacle lenses showed moderate myopia control effects. Progressive addition spectacle lenses, bifocal spectacle lenses, and peripheral defocus modifying spectacle lenses showed weak myopia control effects. In general, children with high accommodative lag and/or near-point esophoria appeared to benefit most from these interventions. The myopia control effect of bifocal and progressive addition spectacle lenses is thought to be based on reducing accommodative effort or accommodative error [7].
Under corrected single vision spectacle lenses were ineffective since They appeared to rather stimulate than prevent myopia progression.

Except for prismatic bifocal spectacle lenses, clinically meaningful slowing of myopia progression by using either bifocal or progressive addition spectacle lenses was not enough to recommend them as routine prescriptions for myopic children [7].

A summary of the different spectacle lens related treatment outcomes is provided in table 2 .

It is noteworthy, that peripheral defocus modifying spectacle lenses were only made for the clinical study and are not available in patient care [9].

\begin{tabular}{|c|c|c|c|}
\hline Intervention & $\begin{array}{c}\text { Refraction } \\
\text { change }(95 \% \text { CI })\end{array}$ & $\begin{array}{c}\text { Axial length } \\
\text { change (95\% CI) }\end{array}$ & $\begin{array}{c}\text { Myopia control } \\
\text { effect }\end{array}$ \\
\hline Prismatic bifocal spectacle lenses & $0.34 \mathrm{D}(0.22$ to 0.46$)$ & $-0.09 \mathrm{~mm}(-0.14$ to -0.04$)$ & Moderate \\
\hline Bifocal spectacle lenses & $0.09 \mathrm{D}(0.05$ to 0.24$)$ & $-0.06 \mathrm{~mm}(-0.10$ to -0.02$)$ & Weak \\
\hline Peripheral defocus modifying spectacle lenses & $0.12 \mathrm{D}(0.06$ to 0.30$)$ & $-0.05 \mathrm{~mm}(-0.12$ to 0.02$)$ & Weak \\
\hline Progressive addition spectacle lenses & $0.12 \mathrm{D}(0.07$ to 0.18$)$ & $-0.04 \mathrm{~mm}(-0.07$ to -0.01$)$ & Weak \\
\hline Undercorrected single vision spectacle lenses & $-0.11 \mathrm{D}(-0.22$ to 0.00$)$ & $0.03(-0.02$ to 0.08$)$ & Ineffective \\
\hline
\end{tabular}

Table 2: Spectacle lens modalities for myopia control compared to single vision lenses or placebo treatment, from most to least effective with respect to axial length change.

\section{Contact Lenses}

Contact lenses used for myopia control have substantial differences in surface geometry, optical design, material, and wearing modalities. This involves single vision RGP contact lenses, single vision soft contact lenses, peripheral defocus modifying soft contact lenses, and RGP contact lenses used for orthokeratology.

Among these modalities, overnight orthokeratology, which involves wearing the lenses during sleep and removing them throughout the day, demonstrated the most effective myopia control. However, a number of issues limit the widespread use of orthokeratology, such as cost, a relatively complicated fitting regimen, and a possibly higher risk of infective keratitis $[10,11]$.

Both orthokeratology as well as wearing peripheral defocus modifying soft contact lenses induce various amounts of myopic defocusing in the retinal periphery, while correcting refractive errors centrally. These refractive profiles are considered key fac- tors to inhibit ocular elongation, myopia development and progression. According to this theory, an eye grows in axial length to bring a hyperopic defocus in its retinal periphery into focus, independently of its foveal refractive status. Several studies have shown that myopic eyes typically have relatively hyperopic peripheral refractions, whereas emmetropic and hyperopic eyes have relatively myopic peripheral refractions. The authors suggested that peripheral hyperopic defocus could be a cause rather than a result of myopia development, and proposed that inducing myopic peripheral defocusing in myopic eyes might be desirable for controlling myopia progression. [12-16].

When evaluating their potential for myopia control, both orthokeratology as well as peripheral defocus modifying soft lenses proved to be moderately effective. Single vision RGP contact lenses and single vision soft lenses, which typically do not induce myopic defocusing in the retinal periphery, were ineffective in slowing the progression of myopia. (Table 3 ). 


\begin{tabular}{|c|c|c|c|}
\hline \multicolumn{1}{|c|}{ Intervention } & $\begin{array}{c}\text { Refraction } \\
\text { change (95\% CI) }\end{array}$ & $\begin{array}{c}\text { Axial length } \\
\text { change (95\% CI) }\end{array}$ & $\begin{array}{c}\text { Myopia control } \\
\text { effect }\end{array}$ \\
\hline Orthokeratology & N/A & -0.14 mm (-0.19 to -0.10$)$ \\
\hline Peripheral defocus modifying soft contact lenses & $0.31 \mathrm{D}(0.02$ to 0.60$)$ & $-0.12 \mathrm{~mm}(-0.19$ to -0.05$)$ & Moderate \\
\hline Single vision soft contact lenses & $-0.06 \mathrm{D}(-0.10$ to -0.02$)$ & $0.01 \mathrm{~mm}(-0.01$ to 0.03$)$ & Ineffective \\
\hline Single vision rigid gas-permeable lenses & $-0.03 \mathrm{D}(-0.13$ to 0.07$)$ & $0.02 \mathrm{~mm}(-0.04$ to 0.08$)$ & Ineffective \\
\hline
\end{tabular}

Table 3: Myopia control interventions involving contact lenses, compared to single vision lenses or placebo treatment, from most to least effective with respect to axial length change.

\section{Pharmacologic Agents}

Pharmacologic interventions may include atropine in various dosages, cyclopentolate, and pirenzepine. In the analyzed RCTs, all of them were administered daily at bedtime. While atropine and cyclopentolate are available as eye drops, pirenzepine is an ophthalmic gel. Timolol, a beta-blocker, was ineffective in controlling myopia progression and should not be used for this purpose. Table 4 summarizes the efficacy of these pharmacologic interventions.

\begin{tabular}{|c|c|c|c|}
\hline Intervention & $\begin{array}{c}\text { Refraction } \\
\text { change (95\% CI) }\end{array}$ & $\begin{array}{c}\text { Axial length } \\
\text { change (95\% CI) }\end{array}$ & $\begin{array}{c}\text { Myopia control } \\
\text { effect }\end{array}$ \\
\hline High-dose atropine (1\% or 0.5\%) & $0.68 \mathrm{D}(0.52$ to 0.84$)$ & $-0.21 \mathrm{~mm}(-0.28$ to -0.16$)$ & Strong \\
\hline Moderate-dose atropine (0.1\%) & $0.53 \mathrm{D}(0.28$ to 0.77$)$ & $-0.21 \mathrm{~mm}(-0.32$ to -0.12$)$ & Strong \\
\hline Low-dose atropine (0.01\%) & $0.53 \mathrm{D}(0.21$ to 0.85$)$ & $-0.15 \mathrm{~mm}(-0.25$ to -0.05$)$ & Strong \\
\hline Cyclopentolate (1\%) & $0.33 \mathrm{D}(0.07$ to 0.59$)$ & Moderate \\
\hline Pirenzepine (2\%) & $0.29 \mathrm{D}(0.05$ to 0.52$)$ & $-0.09 \mathrm{~mm}(-0.17$ to -0.01$)$ & Moderate \\
\hline Timolol (0.25\%) & $-0.02 \mathrm{D}(-0.31$ to 0.27$)$ & N/A & Ineffective \\
\hline
\end{tabular}

Table 4: Myopia control interventions involving pharmacologic agents, compared to single vision lenses or placebo treatment, from most to least effective with respect to refraction and axial length changes.

Atropine, a nonspecific muscarinic receptor antagonist, was the most potent intervention strategy for controlling myopia progression during the time of treatment. In general, the higher the atropine dose (up to $1 \%$ ), the more effective was the myopia control. However, ocular side effects of applying high-dose atropine include pupil dilation, light sensitivity, and near blur $[17,18]$. These effects need to be addressed with spectacle lenses, for instance photochromic, progressive addition spectacle lenses. Furthermore, there was a remarkable myopia rebound effect after cessation of high-dose atropine treatment [19].

When directly comparing the myopia control effect of $0.5 \%$, $0.1 \%$, and $0.01 \%$ atropine over a period of five years, the authors of one RCT found a dose-related response with a greater effect in higher doses during active treatment, but an inverse dose-related increase in myopia during the washout period. In addition, low-dose atropine also caused minimal pupil dilation, minimal loss of accommodation, and no near visual loss. They concluded that over a longer period of time, low-dose atropine $(0.01 \%)$ was more effective in myopia control with less visual side effects compared with higher doses of atropine [20].

This makes high or moderate doses of atropine less likely to be considered as top choices for myopia control interventions in clinical practice. Although the side effects of atropine seem to prevent eye care practitioners from routinely prescribing it for 
myopia control, low-dose atropine may provide adequate myopia control with remarkably fewer side effects and a reduced rate of myopia rebound after discontinuation of the treatment.

Atropine initially was selected for myopia control based on the assumption that accommodation was the causative factor in myopia progression, and therefore cycloplegia may help its control. Currently, there is increasing evidence that the mode of action by which atropine prevents myopia is via interaction with retinal muscarinic receptors, although the evidence is not yet conclusive $[21,22]$.

Cyclopentolate, a nonspecific muscarinic receptor antagonist, was found to be moderately effective in treating myopia progression in an older study [23]. It has atropine-like ocular side effects and does not have a role for myopia control in clinical practice in the United States.

Pirenzepine, an M1-specific muscarinic receptor antagonist, is a possible alternative to low-dose atropine. M1 receptors are abundantly concentrated in the retina and sparsely distributed on ciliary body and iris. Pirenzepine demonstrated a moderate myopia control effect, while only minimally influencing pupil dilation and accommodation, and having a reduced likelihood of causing any atropine-like side effects. Although an effective myopia control agent, pirenzepine does not currently have a role in clinical practice in the United States, since it is not commercially available in an ophthalmic preparation [7].

\section{More Outdoor Activities}

Some studies have reported that children and adolescents with more outdoor activities tend to have a reduced myopia progression rate and suggested that outdoor activities were protective for myopia development and progression. One meta-analysis of seven cross-sectional studies reported a significant protective association between increasing time spent outdoors and prevalent myopia in children and adolescents. Each increase in hours per week of time spent outdoors was associated with a $2 \%$ reduced odds of myopia, after adjustment for potential confounders [6].

Instead of using cross-sectional data, one RCT, reports a weak myopia control effect for more outdoor activities of 14 to 15 hours per week. The associated mean change in refraction over one year was $0.14 \mathrm{D}$ (95\% CI: $0.06-0.22)$, which is considered a weak effect. No information regarding changes in axial lengths of the eyes was available [24].

Other studies report that increased time outdoors was effective in preventing the onset of myopia as well as subsequently slowing the myopic shift in refractive error. However, outdoor time was not effective in slowing progression in eyes that were already myopic [25].

Therefore, increasing time spent outdoors may be a simple strategy to reduce the risk of developing myopia in the first instance and allow for a weak control effect in its subsequent progression.

\section{Conclusion}

A meta-analysis of myopia control RCTs indicates that atropine at different dosages was superior to any other interventions. The higher the atropine dose, the better the control effect during the time of treatment. Disadvantages of higher dose atropine $(1 \%$, $0.5 \%$, and $0.1 \%$ ) were side effects and accelerated myopia progression after discontinuation of treatment. Low-dose atropine $(0.01 \%)$ however provided adequate myopia control with remarkably fewer side effects and reduced myopia rebound effect. Additionally, over a longer period of time, low-dose atropine was found to be more effective in myopia control compared to higher doses of atropine, which makes it a potentially better choice in clinical practice. In the United States, atropine doses other than $1 \%$ are only available through compounding pharmacies.

Cyclopentolate and pirenzepine provided comparable, moderate levels of myopia control. While cyclopentolate has a number of side effects, pirenzepine is not readily available as an ophthalmic preparation. Therefore, these pharmaceutical agents are currently not used for myopia control.

Orthokeratology, as well as peripheral defocus modifying soft contact lenses demonstrated comparable, moderate myopia control effects. Since the latter are soft contact lenses, their fitting regimen is much less complex as well as less expensive compared to orthokeratology, which renders them an attractive option in clinical practice. In recent years, this lens modality got a good amount of interest, which sparked a variety of design modifications. Currently, several clinical studies are underway, assessing the myopia control potential of these new generation lenses.

For patients who neither will be treated with pharmacologic agents nor with contact lenses, prismatic bifocal spectacle lenses are an option, providing a moderate level of myopia control. Especially when used in young and adolescent patients, the target group for myopia control, cosmesis with these lenses might be problematic though, as well as the permanent presence of the add power segment in the lower half of the lenses.

Increased time spent outdoors was effective in preventing the onset of myopia and showed a weak myopia control potential, once myopia was established. It therefore can be recommended as a simple strategy to reduce the risk of developing myopia and its progression in children and adolescents.

Progressive addition spectacle lenses and bifocal spectacle lenses only produced weak myopia control effects and therefore cannot be recommended as first choice treatment options. Similar weak effects were demonstrated by peripheral defocus modifying spectacle lenses, which are not available in clinical practice.

Undercorrected single vision spectacle lenses, single vision soft contact lenses, single vision RGP contact lenses in alignment fit, and administration of timolol eye drops were ineffective in controlling myopia progression and should not be considered as 
treatment options.It must be noted, that no intervention is currently approved for myopia control by the United States Food and Drug Administration. Therefore all discussed interventions can only be used off-label and patients should be informed about this before considering treatment. Since the prevalence of myopia is rising, new or modified treatment options are necessary. Therefore more RCTs are needed to further confirm the myopia control effect by different interventions.

\section{References}

1. Saw SM, Shih-Yen EC, Koh A, Tan D (2002) Interventions to retard myopia progression in children: an evidence-based update. Ophthalmology 109: 415-421.

2. Vitale S, Sperduto RD, Ferris FL (2009) Increased prevalence of myopia in the United States between 1971-1972 and 1999-2004. Arch Ophthalmol 127: 1632-1639.

3. Ding BY, Shih YF, Lin LLK, Hsiao CK, Wang IJ (2017) Myopia among schoolchildren in East Asia and Singapore. Surv Ophthalmol 62: 677-697.

4. Holden BA, Fricke TR, Wilson DA, Jong M, Naidoo KS, et al. (2016) Global Prevalence of Myopia and High Myopia and Temporal Trends from 2000 through 2050. Ophthalmology 123:1036-1042.

5. Morgan IG, French AN, Ashby RS, Guo X, Ding X, et al. (2017) The epidemics of myopia: Aetiology and prevention. ProgRetin Eye Res 1350-9462: 30039-30043.

6. Sherwin JC, Reacher MH, Keogh RH, Khawaja AP, Mackey DA, et al. (2012) The association between time spent outdoors and myopia in children and adolescents: a systematic review and meta-analysis. Ophthalmology 119: 2141-2151.

7. Walline JJ (2016) Myopia Control: A Review. Eye Contact Lens 42: 3-8.

8. Huang J, Wen D, Wang Q, McAlinden C, Flitcroft I, et al. (2016)Efficacy Comparison of 16 Interventions for Myopia Control in Children: A Network Meta-analysis. Ophthalmology 123: 697-708.

9. Sankaridurg P, Donovan L, Varnas S, Ho A, Chen X, et al. (2010) Spectacle lenses designed to reduce progression of myopia: 12-month results. Optom Vis Sci 87: 631-641.

10. Cho P, Cheung SW, Edwards MH, Fung J (2003) An assessment of consecutively presenting orthokeratology patients in a Hong Kong based private practice. Clin Exp Optom 86: 331-338.

11. Chan TC, Li EY, Wong VW, Jhanji V (2014) Orthokeratology-associated infectious keratitis in a tertiary care eye hospital in Hong Kong. Am J Ophthalmol 158: 1130-1135.
12. Kang $P$, Swarbrick $H$ (2011) Peripheral refraction in myopic children wearing orthokeratology and gas-permeable lenses. Optom Vis Sci 88: 476-482.

13. Wallman J, Winawer $\mathrm{J}$ (2004) Homeostasis of eye growth and the question of myopia. Neuron 43: 447-468.

14. Charman WN, Radhakrishnan (2010) H Peripheral refraction and the development of refractive error: a review. Ophthalmic Physiol Opt 30: 321-338.

15. Charman WN, Mountford J, Atchison DA, Markwell EL (2006) Peripheral refraction in orthokeratology patients. Optom Vis Sci 83: 641-648.

16. Berntsen DA, Kramer CE (2013) Peripheral defocus with spherical and multifocal soft contact lenses. Optom Vis Sci 90: 1215-1224.

17. Kennedy RH, Dyer JA, Kennedy MA, Parulkar S, Kurland LT, et al. (2000)Reducing the progression of myopia with atropine: a long term cohort study of Olmsted County students. Binocul Vis Strabismus Q 15: 281-304.

18. Gong Q, Janowski M, Luo M, Wei H, Chen B, et al. (2017)Efficacy and Adverse Effects of Atropine in Childhood Myopia: A Meta-analysis. JAMA Ophthalmol 135: 624-630.

19. Tong L, Huang XL, Koh AL, Zhang X, Tan DT, et al. (2009) Atropine for the treatment of childhood myopia: effect on myopia progression after cessation of atropine. Ophthalmology 116: 572-579.

20. Chia A, Lu QS, Tan D (2016) Five-Year Clinical Trial on Atropine for the Treatment of Myopia 2: Myopia Control with Atropine $0.01 \%$ Eyedrops. Ophthalmology 123: 391-399.

21. Pineles SL, Kraker RT, VanderVeen DK, Hutchinson AK, Galvin JA, et al. (2017)Atropine for the Prevention of Myopia Progression in Children: A Report by the American Academy of Ophthalmology. Ophthalmology 124: 1857-1866.

22. McBrien NA, Stell WK, Carr B (2013) How does atropine exert its anti-myopia effects? Ophthalmic Physiol Opt 33: 373-378.

23. Yen MY, Liu JH, Kao SC, Shiao CH (1989) Comparison of the effect of atropine and cyclopentolate on myopia. Ann Ophthalmol 21:180182.

24. Yi JH, Li RR (2011) Influence of near-work and outdoor activities on myopia progression in school children. Zhongguo Dang Dai ErKeZaZhi 13: 32-35.

25. Xiong S, Sankaridurg P, Naduvilath T, Zang J, Zou H, et al. (2017) Time spent in outdoor activities in relation to myopia prevention and control: a meta-analysis and systematic review. Acta Ophthalmologica95: 551-566. 